\title{
Vertical excitation of axisymmetric liquid bridges
}

\author{
J.-B. Valsamis ${ }^{\mathrm{a}, *}$, M. Mastrangeli $^{\mathrm{b}}$, P. Lambert ${ }^{\mathrm{a}}$ \\ a BEAMS Department CP 165/56, Université Libre de Bruxelles, Avenue F. D. Roosevelt 50, B-1050 Bruxelles, Belgium \\ ${ }^{\mathrm{b}}$ Ecole Polytechnique Fédérale de Lausanne (EPFL), CH-1015 Lausanne, Switzerland
}

\section{A R T I C L E I N F O}

\section{Article history:}

Received 29 May 2012

Accepted 28 September 2012

Available online 17 October 2012

\section{Keywords:}

Microfluidics

Mechanical model

Free interface

Kelvin-Voigt system

Vertical oscillation

\begin{abstract}
A B S T R A C T
This study presents an analytical model of the dynamics of an axisymmetric liquid bridge confined between two circular pads and subjected to small vertical periodic perturbations. Such system finds important applications in microassembly and microjoint design, where force and damping need to be precisely controlled. The liquid bridge is modelled by an equivalent spring/dashpot/mass system characterised by the spring constant $k$, the damping coefficient $b$ and the equivalent mass $m$, respectively. An abacus for $k$ as well as analytical approximations for $k, b$ and $m$ based on simplifications of the Navier-Stokes equation are provided. The study is validated by experiments and numerical simulations of the system. We describe the experimental setup we designed to investigate vertical forces arising on the bottom pad from small periodic perturbations of the top pad confining the liquid meniscus. The setup allowed the accurate control of all physical and geometrical parameters relevant for the experiments. The parameters we investigated are both physical (viscosity and surface tension of the fluid) and geometrical (the edge angle between the meniscus and the pad, the height of the meniscus). The good agreement between model predictions and results let us conclude that $k, b$ and $m$ involve only one physical property of the liquid, namely the surface tension, the viscosity and the density, respectively.
\end{abstract}

(c) 2012 Elsevier Masson SAS. All rights reserved.

\section{Introduction}

Liquid bridges are liquid volumes confined between two solid surfaces and surrounded by another fluid (most commonly, air). Mechanically, liquid bridges can be considered as joints between two solids (e.g. a substrate and a component). Upon perturbation, they generate capillary forces that can be repulsive or attractive depending on several properties of the fluid and the bounding solids. These properties are both geometrical and physical [1-3].

Forces generated by liquid bridges are ubiquitous, and technologically relevant for e.g. flip-chip electronic assembly [4-6] and capillary self-assembly of micro- and nanosystems [7,8]. Analytical models and quasi-static numerical simulations - typically performed through SURFACE EVOLVER [9] - for such applications are widely reported $[3,10]$. Different degrees of freedom are thereby strained, and the restoring forces or torques are computed. Although fully three-dimensional, these numerical models do not contemplate dynamics.

Dynamical studies were introduced by van Veen [11] and Meurisse and Querry [12]. They proposed analytical models for

\footnotetext{
* Corresponding author.

E-mail address: jvalsami@ulb.ac.be (J.-B. Valsamis).
}

dynamical parameters and restoring forces, and for the pressure of the liquid bridge as a function of the inverse of its gap, respectively. Cheneler et al. proposed an analysis of a liquid bridge to develop a micro-rheometer [13]. The liquid is modelled as a dashpot in series with a calibrated spring/mass system. By measuring the phase shift between the position of the mass and the force exerted, the friction can be deduced. However, inertial effects are not included in the model, and the study is not supported with experimental data.

Engmann et al. [14] presented a comprehensive review of main analytical expressions of viscous force corresponding to several squeeze film configurations, e.g. through slipping, nonslipping, viscoelastic, viscoplastic. In particular, the expressions of the viscous term developed by Pitois et al. [15] can thereby be recovered.

Concerning numerical simulations, Boufercha et al. [16] estimated the time response and the position error of a selfpositioning process of a chip on a substrate. The simulation includes the motion of a liquid drop crushing a substrate made of hydrophobic and hydrophilic regions, and the squeezing of the drop by the chip. Lu and Bailey [17] devised a model to determine the timescale of a chip self-alignment process. Their approach consists in coupling the motion of the solder driven by the chip, and of the chip itself. The coupling is justified by the identical timescales of the motion of both elements. They concluded that the usual, uncoupled model underestimates the impact of viscosity. 


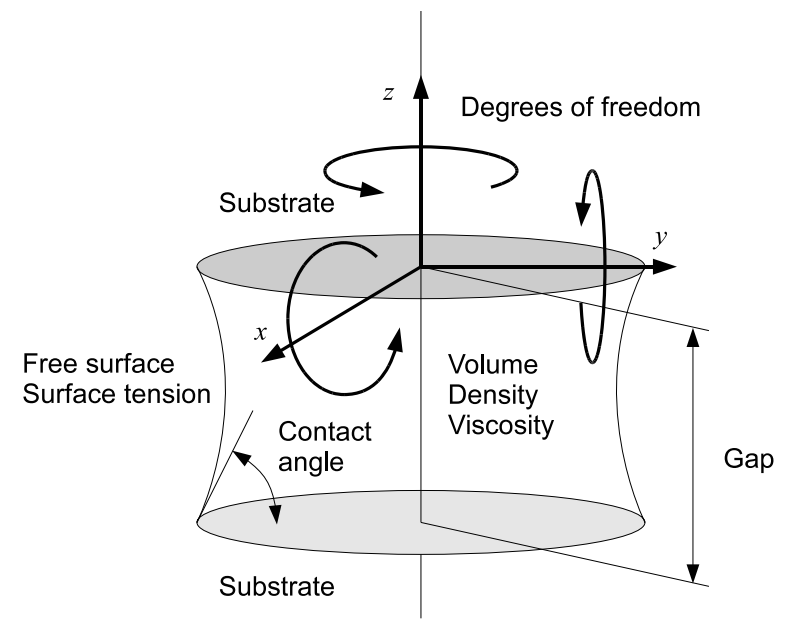

Fig. 1. Degrees of freedom for a liquid bridge between a couple of solid interfaces. The substrates refer to generic solid surfaces. They can belong to a couple made of e.g. a gripper (top) and a component (bottom), or of a component (top) and a substrate (bottom).

Other problems involving the free liquid surface were addressed in a rather different way by Montanero $[18,19]$. He studied the possible oscillations of an air/liquid interface. In this case, the deformation is due to the inertia of the fluid, and fluids of low viscosity are required. Beyond the theoretical consideration of inertial effect, the vibration of the interface loses interest in the problem addressed in the present work. As it will be shown, the effect of the capillary force is completely negligible at frequencies for which the shape of the free interface presents wavelets. Finally, dynamical studies of lateral forces of liquid bridges were conducted by Lambert et al. [20], including the effect of viscosity as well as free surface forces.

In this paper, we consider an axisymmetric liquid bridge periodically excited along its vertical axis. The dynamic behaviour of the liquid bridge is modelled by an equivalent spring/dashpot/mass system characterised by the spring constant $k$, the damping coefficient $b$ and the mass $m$. The study provides an abacus and analytical approximations (by means of both a parabolic and a circular model) for $k$, as well as analytical laws for $b$ and $m$. The analytical predictions are compared with experiments and numerical simulations. The good agreement obtained allows us to confirm the assumptions of the model, described in the following section.

\section{Liquid bridge model}

When the substrate (bottom solid) is fixed, the component (top solid) standing on top of the liquid bridge has six degrees of freedom with reference to the substrate: three translational and three rotational (Fig. 1). For small periodic perturbations along the degrees of freedom, the dynamic response of these degrees of freedom may be decoupled into six frequential responses. The responses can be described in several ways, depending on how the system is excited (the input) on one hand, and on how the effect of the excitation (the output) is passed on, on the other.

This study focuses on the translational degree of freedom along $z$ (defined in Fig. 1). The liquid bridge is pinned by two circular and parallel interfaces providing an axially-symmetric geometry around the $z$ axis. All liquid bridge dimensions are smaller than the liquid capillary length.

\subsection{Edge angle}

As described later, the bounding plates of our experimental setup present sharp edges to ensure the pinning of the liquid.

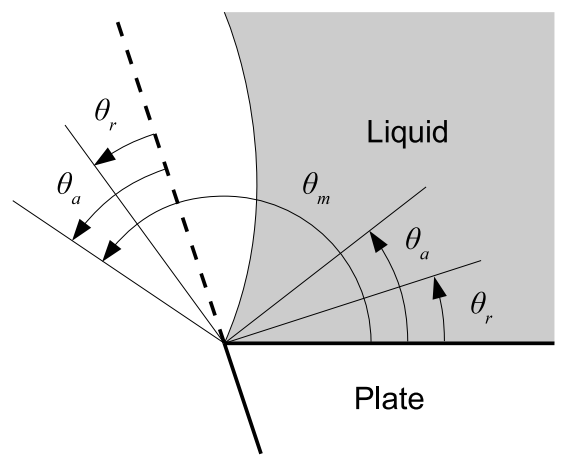

Fig. 2. Pinning of the triple contact line. When the liquid is pinned on a sharp edge, the edge angle can be higher than the advancing contact angle.

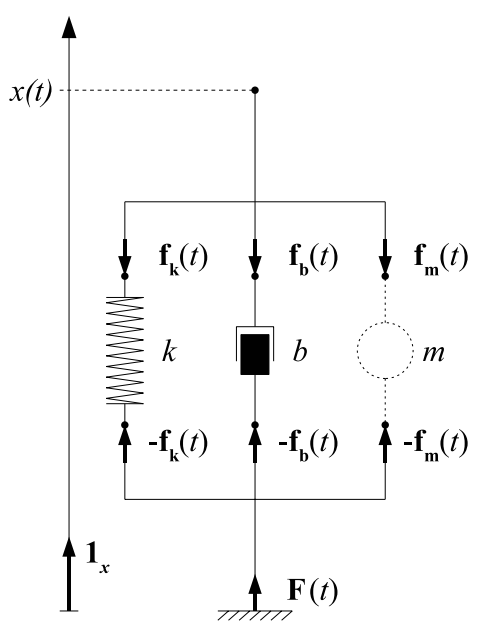

Fig. 3. The Kelvin-Voigt model. Forces $f_{k}(t)$ and $f_{b}(t)$ represent the force of the spring and of the dashpot, respectively, while $f(t)$ represents the total force exerted by liquid bridge on the system. Origin is assumed at the free length position. The direction of forces are represented for a stretched spring $x(t)>0$, upwards velocity $\dot{x}(t)>0$ and upwards acceleration $\ddot{x}(t)>0$.

Liquid pinning avoids the motion of the triple contact line, and affords the advantage of a less constrained edge angle. Indeed, considering a planar surface, it is known that the liquid will recede if its contact angle is smaller than the receding angle $\theta_{r}$ and will advance if higher than the advancing angle $\theta_{a}$. The plates are described by two surfaces - one horizontal in contact with the bridge, the other nearly vertical defining the edge - on which receding and advancing angles are considered (as shown in Fig. 2 for the bottom plate). In this case, the liquid will recede if the contact angle with the horizontal surface is smaller than $\theta_{r}$, and will advance only if the contact angle on the edge surface is higher than $\theta_{a}$. Considering the horizontal plane as reference, the liquid will remain as long as the contact is between $\theta_{r}$ and $\theta_{m}$ Fig. 2. Moreover, liquid pinning simplifies the problem because, by avoiding the triple contact line motion, the no slip condition applies.

\subsection{Model}

The mechanical model of the axial degree of freedom is presented in Fig. 3: a Kelvin-Voigt system made up of a spring (of stiffness $k$ ), a damper dashpot (of damping coefficient $b$ ) and an equivalent inertial force (of mass $m$ ) connected in parallel. Such a system can be described by its frequential response and is entirely defined when the coefficients $k, b$ and $m$ are known.

Typical input/output pairs are the position of an interface, its velocity, its acceleration and its force. The system can be completely 
described by the frequential response of a single input/output pair. Here, we will characterise the vertical translation considering as input the displacement of the top interface $x(t)$, and as output the force exerted on the bottom interface $F(t)$.

The mass appears through inertial effects, which include acceleration. As a consequence, from a sufficiently-high excitation frequency onward, inertial effects cannot be neglected.

The gravitational effects can conversely be ignored because the dimensions involved (750 $\mu \mathrm{m}$ for the radius, around $200 \mu \mathrm{m}$ for the gap) are below the capillary length $\left(L_{c}=\sqrt{\frac{\gamma}{\rho g}} \approx 1.4 \mathrm{~mm}\right.$ for the liquids used, see Section 4).

The expression of the forces is:

$\mathbf{f}_{\mathbf{k}}(t)=-k x(t) \mathbf{1}_{x}$

$\mathbf{f}_{\mathbf{b}}(t)=-b \dot{x}(t) \mathbf{1}_{x}$

$\mathbf{f}_{\mathbf{m}}(t)=-m \ddot{x}(t) \mathbf{1}_{x}$.

The force exerted by the meniscus on the lower interface is thus:

$\mathbf{F}(t)=-\mathbf{f}_{\mathbf{k}}(t)-\mathbf{f}_{\mathbf{b}}(t)-\mathbf{f}_{\mathbf{m}}(t)$.

With periodic input of pulse $\omega$, the system can be rewritten with phasors giving a transfer function of gain $G$ and phase $\phi$ :

$$
\begin{aligned}
& G(\omega)=\sqrt{\left(k-\omega^{2} m\right)^{2}+\omega^{2} b^{2}} \\
& \phi(\omega)=\arctan \frac{\omega b}{k-\omega^{2} m} .
\end{aligned}
$$

\section{Analytical estimation of coefficients}

We present an analytical development to approximate the coefficients of the Kelvin-Voigt model describing the liquid bridge. The aim is to decouple the liquid properties, namely the surface tension, the viscosity and the density, into the stiffness $k$, the damping $b$ and the inertial mass $m$, respectively.

Briefly, the spring force considers the fluid at rest, the damping force considers only the viscous force driving the fluid, and the inertial force considers the force due to fluid acceleration. The Navier-Stokes equation is simplified consequently. The force applied by the fluid on the bottom interface is then computed, and the corresponding coefficients are derived. Gravitational effects are ignored, while inertial ones are contemplated where required.

\subsection{Stiffness $k$}

The force generated by a spring is independent of the speed at which its extremity moves. Therefore, to calculate the stiffness, it is appropriate to consider the liquid at rest. The pressure outside the liquid bridge $p_{0}$ is assumed to be zero.

The geometry and the parameters are presented in Fig. 4. In addition to the axial symmetry, the geometry presents a symmetry with respect to the $r$ axis, which further reduces the parameters in the study. The input parameters are the radius of the plate $r_{b}$, the gap (or meniscus height) $h$, and the edge angle $\theta$. By fixing these three parameters, the meniscus volume is automatically determined.

The stiffness $k$ can be deduced from the derivative of the spring force $f_{k}$. Without damping nor inertial effect and using (1) and (4):

$k=-\frac{\mathrm{d} f_{k}}{\mathrm{~d} h}=\frac{\mathrm{d} F}{\mathrm{~d} h}$.

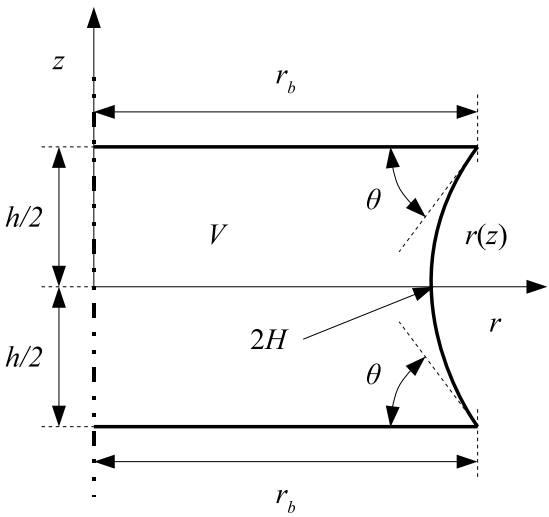

Fig. 4. Geometry and parameters for the analytical model

Since the liquid is at rest, the inner pressure is due to the curvature of the free interface ${ }^{1}$.

$p=2 H \gamma$.

The force of the liquid is thus the sum of the Laplace and the surface tension forces:

$F=2 \pi \gamma r_{b} \sin \theta-2 \pi \gamma r_{b}^{2} H$.

And the stiffness is:

$k=\frac{\mathrm{d} F}{\mathrm{~d} h}=2 \pi \gamma r_{b} \cos \theta \frac{\mathrm{d} \theta}{\mathrm{d} h}-\pi \gamma r_{b}^{2} \frac{\mathrm{d}(2 H)}{\mathrm{d} h}$.

The derivatives are done assuming the volume is constant. With the same convention ${ }^{1}$, the local curvature of an analytical axisymmetric shape $r(z)$ is:

$2 H(z)=-\frac{r^{\prime \prime}(z)}{\left[1+r^{\prime 2}(z)\right]^{\frac{3}{2}}}+\frac{1}{r(z) \sqrt{1+r^{\prime 2}(z)}}$.

Unfortunately, there is no analytical solution $r(z)$. Hence, we adopt the following three strategies:

1. Calculating numerically the shape.

2. Considering a parabolic shape and.

3. Assuming a circular shape.

The first method is the numerical integration of (11) that matches with boundary conditions. ${ }^{2}$ The derivative of the curvature and the edge angle is computed by the finite difference method.

The parabolic and circular models provide analytical relations. Yet, they do not have a constant curvature on the whole interface since they are not the solution of (11). The curvature and its derivative are taken at the neck of the meniscus $(z=0)$. These relations are summarised in Table 1.

\subsection{Damping coefficient $b$}

The evaluation of the damping coefficient highlights the effect of the viscosity of the fluid. This state is characterised by a low Reynolds number. We assume the surface tension to have a negligible impact compared to the viscous force; in particular, the variation of pressure due to the surface tension is negligible compared to the variation of pressure due to viscous forces. The acceleration term is also neglected, so that the Navier-Stokes equation contains only pressure and viscous terms.

\footnotetext{
1 The sign of the curvature depends on the direction of the normal vector. The convention hereby adopted has the normal pointing outside of the liquid, giving a positive curvature for a sphere.

2 We used bvp4c in MatLaB $\left.{ }^{(}\right)$
} 
Table 1

Analytical expressions from the parabolic model and the circular model.

\begin{tabular}{l}
\hline Parabolic model \\
$\frac{\mathrm{d} \theta}{\mathrm{d} h}=-\sin \theta \frac{30 r_{b}^{2} \sin ^{2} \theta-20 r_{b} h \sin \theta \cos \theta+3 h^{2} \cos ^{2} \theta}{10 r_{b} h^{2} \sin \theta-2 h^{3} \cos \theta}$ \\
$\frac{\mathrm{d} 2 H}{\mathrm{~d} h}=2 \cot \theta\left(\frac{1}{h^{2}}+\frac{2}{\left(4 r_{b}-h \cot \theta\right)^{2}}\right)+\frac{2 h}{\sin ^{2} \theta}\left(\frac{1}{h^{2}}-\frac{2}{\left(4 r_{b}-h \cot \theta\right)^{2}}\right) \frac{\mathrm{d} \theta}{\mathrm{d} h}$ \\
\hline Circular model \\
$\frac{\mathrm{d} \theta}{\mathrm{d} h}=-\frac{4 r_{b}^{2} \cos ^{4} \theta+4 r_{b} h \sin \theta \cos ^{3} \theta+3 h^{2} \cos ^{2} \theta-h^{2} \cos ^{4} \theta}{3 h^{3} \sin \theta \cos \theta+4 r_{b} h^{2} \cos ^{2} \theta+\left(\frac{\pi}{2}-\theta\right)\left(2 h^{3} \cos ^{2} \theta-3 h^{3}-2 r_{b} h \sin 2 \theta\right)}-$ \\
$\frac{-\left(\frac{\pi}{2}-\theta\right)\left(3 h^{2} \sin \theta+4 r_{b} h \cos \theta\right)}{3 h^{3} \sin \theta \cos \theta+4 r_{b} h^{2} \cos ^{2} \theta+\left(\frac{\pi}{2}-\theta\right)\left(2 h^{3} \cos ^{2} \theta-3 h^{3}-2 r_{b} h \sin 2 \theta\right)}$ \\
$\frac{\mathrm{d} 2 H}{\mathrm{~d} h}=\frac{2 \cos \theta}{h^{2}}+\frac{2 \sin \theta}{h} \frac{\mathrm{d} \theta}{\mathrm{d} h}+\frac{1-\sin \theta}{\left(2 r_{b} \cos \theta-h(1-\sin \theta)\right)^{2}}\left(2 \cos \theta-2 h \frac{\mathrm{d} \theta}{\mathrm{d} h}\right)$
\end{tabular}

The damping coefficient $b$ can be deduced from the derivative of the damper force $f_{b}$. Without spring nor inertial effect and using (2) and (4):

$b=-\frac{\mathrm{d} f_{b}}{\mathrm{~d} \dot{h}}=\frac{\mathrm{d} F}{\mathrm{~d} \dot{h}}$.

The considered geometry is cylindrical. The parameters are identical to the approximation of the stiffness (Fig. 4), except for the edge angle which is $\theta=90^{\circ}$. Although a velocity field appears in the meniscus, the deformation induced is small enough to be ignored, so that the geometry can be considered constant.

The force applied by the liquid on the bottom plate is defined by the sum of all the constrains on the plate:

$$
\begin{aligned}
F \mathbf{1}_{z} & =\left[\int_{\Gamma_{b}}(-p \overline{\overline{\mathrm{I}}}+\overline{\bar{\tau}}) \cdot \mathrm{d} \mathbf{S}\right] \cdot \mathbf{1}_{z} \\
& =\left[\int_{\Gamma_{b}}\left(-p+2 \mu \frac{\partial u_{z}}{\partial z}\right) \mathrm{d} S\right] \mathbf{1}_{z}
\end{aligned}
$$

In the complete review of Engmann et al. [14], the authors propose some assumptions on the velocity profile of a film of liquid squeezed by two parallel plates at constant velocity. The vertical velocity field (i.e. the $z$ component) is assumed to be independent on $r$. Then, the starting assumption reads:

$\mathbf{u}=u_{r}(t, r, z) \mathbf{1}_{r}+u_{z}(t, z) \mathbf{1}_{z}$.

On the fluid/solid interface, the fluid cannot slip. The bottom interface is fixed and the top interface moves at a velocity $\dot{h}(t)$. The boundary conditions of the fluid are:

$u_{z}\left(t,-\frac{h}{2}\right)=0$

$u_{z}\left(t, \frac{h}{2}\right)=\dot{h}(t)$

$u_{r}\left(t, r, \pm \frac{h}{2}\right)=0$.

The cylindrical axisymmetric form of the mass conservation equation and the Navier-Stokes equation gives the following solution inside the meniscus:

$$
\begin{aligned}
\mathbf{u}(t, r, z)= & \dot{h}(t) r\left(3 \frac{z^{2}}{h^{3}}-\frac{3}{4 h}\right) \mathbf{1}_{r} \\
& +\dot{h}(t)\left(-2 \frac{z^{3}}{h^{3}}+\frac{3}{2} \frac{z}{h}+\frac{1}{2}\right) \mathbf{1}_{z} \\
p(t, r, z)= & 3 \mu \dot{h}(t) \frac{r^{2}}{h^{3}}-6 \mu \dot{h}(t) \frac{z^{2}}{h^{3}}+K(t) .
\end{aligned}
$$

Since it is an approximation, the stress is not balanced at the interface. We define the constant $K(t)$ of the pressure field by assuming a zero average stress at the interface:

$\int_{\Gamma_{i}}-p+\left(\overline{\bar{\tau}} \cdot \mathbf{1}_{r}\right) \cdot \mathbf{1}_{r} \mathrm{~d} S=0$.

The force computed on the bottom interface using (14) leads to the damper coefficient:

$b=\frac{3 \pi}{2} \frac{\mu r_{b}^{4}}{h^{3}}+2 \pi \frac{\mu r_{b}^{2}}{h}$.

When the gap is very small compared to the radius $\left(r_{b} \gg h\right)$, the zero average stress is similar to a zero pointwise pressure at the triple line, as used by Engmann. The second term of (22) can then be neglected, and the relation in [14] is recovered.

\subsection{Equivalent mass $m$}

The equivalent mass $m$ can be deduced from the derivative of the inertial force $f_{m}$. Without spring nor damping effect and using (3) and (4):

$m=-\frac{\mathrm{d} f_{m}}{\mathrm{~d} \ddot{h}}=\frac{\mathrm{d} F}{\mathrm{~d} \ddot{h}}$.

The assumption on the geometry is identical to the damping coefficient case (Fig. 4 with $\theta=90^{\circ}$ ).

We assume that the only property governing the fluid is the density. The 2D axisymmetric Navier-Stokes equation will only contain the partial time derivative and the pressure term. For sinusoidal excitation $(h(t)=H \sin 2 \pi f t)$, only the inertial term contains the squared frequency. The convective term is small compared to the acceleration term because the amplitude $H$ of the excitation appears squared (i.e. $H^{2} \ll H$ ).

With increasing inertial effect, the laminar flow changes into a boundary layer. Outside of the layer, the fluid velocity is uniform. Asymptotically the layer thickness tends towards zero and we may assume that the fluid slips on the solid interface. The assumption on the fluid is still (15). With the kinematic of the fluid/solid interfaces, the solution of the simplified axisymmetric equations gives:

$\mathbf{u}(t, r, z)=-\ddot{h}(t) \frac{r}{2 h} \mathbf{1}_{r}+\ddot{h}(t)\left(\frac{z}{h}+\frac{1}{2}\right) \mathbf{1}_{z}$

$p(t, r, z)=\rho \ddot{h}(t)\left(\frac{r^{2}}{4 h}-\frac{z^{2}}{2 h}-\frac{z}{2}\right)+K(t)$.

The expression of the velocity shows that the radial velocity is constant on a cylinder defined by $r=C$ and the axial velocity is constant on a plane $z=C$. The constant $K(t)$ is defined by assuming a zero average stress at the interface, as expressed in (21) and neglecting the viscous stresses. The force exerted on the bottom interface, (14) without the viscous term, gives an equivalent mass:

$m=\pi \rho r_{b}^{2} h\left(\frac{r_{b}^{2}}{8 h^{2}}-\frac{1}{6}\right)$.

\section{Experimental setup}

\subsection{Devices}

The experimental setup is shown in figure Fig. 5(a). A liquid bridge was pinned between two small circular plates (diameter: $1.5 \mathrm{~mm}$, height: $170 \mu \mathrm{m}$ ). The bridge was excited by the motion of the upper plate with a piezoelectric actuator ( $\mathrm{P}-842.40$ Physik 


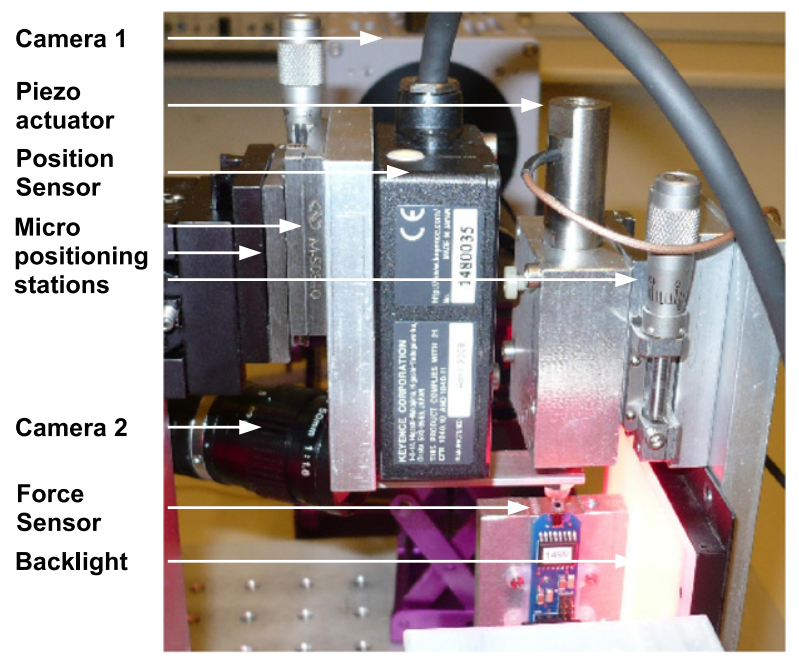

(a) Experimental setup.

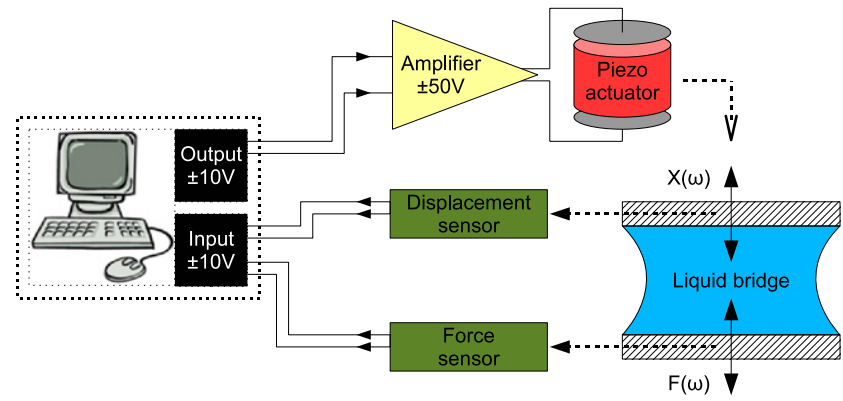

(b) Connection diagram with actuator and sensors.

Fig. 5. Experimental setup used to excite the liquid bridge.

Table 2

Relevant characteristics of silicon oils. DFxxx means DC200FLUIDxxx.

\begin{tabular}{lllll}
\hline Ref & Liquid & Dens. $\mathrm{kg} / \mathrm{m}^{3}$ & Dyn. visc. Pa s & Surf. tens. N/m \\
\hline OIL1 & R47V500 & 970 & 0.485 & $21.110^{-3}$ \\
OIL2 & R47V5000 & 973 & 4.865 & $21.110^{-3}$ \\
OIL6 & DF100 & 960 & 0.096 & $20.910^{-3}$ \\
OIL7 & DF1000 & 971 & 0.971 & $21.210^{-3}$ \\
\hline
\end{tabular}

Instrumente). To accurately measure the displacement of the upper plate, we used a CCD laser displacement sensor (LK-G10 Keyence, controller LK-G3001PV), whose laser beam targeted the top side of the upper plate. A small beam (aluminium alloy, $40 \times$ $14 \times 6.3 \mathrm{~mm}^{3}$ ) was used as mechanical interface to offset the upper plate. The dimension of the beam (especially its height) ensured its first natural frequency $(2875 \mathrm{~Hz},[21])$ to be very far from the excitation frequency of the meniscus, so that the beam could be considered rigid. The force exerted by the liquid bridge on the bottom plate was measured thanks to a highly accurate micro force sensor (FT-S270 Femto Tools). The maximum measurable force was $2000 \mu \mathrm{N}$ with an accuracy of about $5 \mu \mathrm{N}$ (the accuracy was actually dependent on the sampling rate of measurement).

The liquid bridge was filmed by two cameras. Their optical axes made an angle of $70^{\circ}$ (orthogonality was physically not possible on our setup) in order to control the alignment of the two circular plates and the shape of the interface. To ensure the alignment of the plate, the sensor was mounted on a two-directional linear stage (DS40-XY Newport). The whole setup was mounted on a vibration isolating workstation (VH 3030 W-OPT Newport).

Signals generated by both sensors were acquired with a control and measurement platform (NI PXI 1042 with a NI PXI 6723 for analog outputs and a NI PXI 6224 for digital inputs). The analog output was used to drive a home-made amplifier [22] that supplied the piezoelectric actuator. The logical diagram of devices interconnection is shown in Fig. 5(b).

The liquids used in our experiments were silicon oils whose characteristics are given in Table 2 (Rhodia (Rhodorsil Oil) and Dow Corning (DC Fluids)). They had a wide range of viscosities while density and surface tension were almost constant. All the experiments were performed in a laboratory environment: room temperature varied between 24 and $26{ }^{\circ} \mathrm{C}$ and monitored relative air humidity was about $35 \%$.

\subsection{Measurement protocol}

Thanks to the data acquisition system, measurements were almost fully automated. For each experiment, the following steps were adopted:

1. The circular plates were cleaned with acetone and ethanol.

2. A small amount of liquid (around $1 \mu \mathrm{L}$ ) was dropped on the lower plate.

3. The upper plate was lowered until the contact with the liquid bridge.

4. The lower plate was accurately positioned and aligned thanks to the two cameras.

5. The gap was fixed. If the volume needed to be adjusted, the process restarted from step 2.

6. Two pictures were taken in order to compute the volume.

7. Dynamic parameters (frequency range, delays, amplitude of actuator) were defined.

8. A systematic acquisition program in LABVIEW was used:

(a) An output was generated from the output analog signal of the piezoelectric driver.

(b) According to the oil viscosity and the actuation frequency, a delay was inserted to avoid any effect of transient response.

(c) Data were acquired and recorded.

9. Two pictures were taken to control the final state of the meniscus.

The protocol was restarted from step 8 to successively perform multiple experiments on the same system, from step 7 to get the effect of the amplitude of the actuator, from step 5 to change the gap and from step 1 to change the liquid.

\section{Results and discussion}

The methodology adopted to validate the results consists of two steps (see Fig. 6): (i) a comparison between the analytical approximations of the mechanical parameters derived from the Navier-Stokes problem and numerical simulations, and (ii) a comparison between experimental and numerical data. That is, the numerical simulations act like a buffer between analytical expressions and experiments. The first comparison supports the 


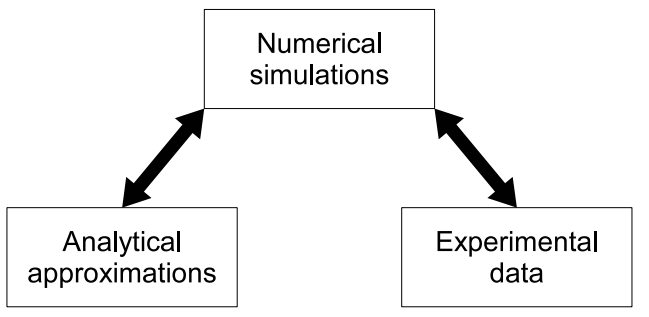

Fig. 6. Link between analytical, numerical and experimental results.

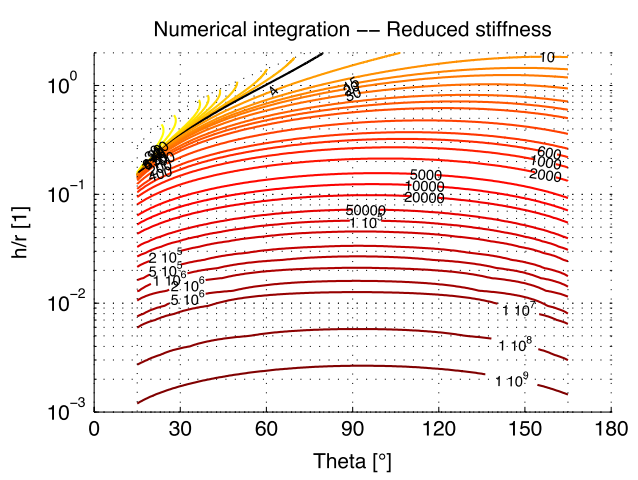

Fig. 7. Map of reduced stiffness $\hat{k}=k / \gamma$ according to the edge angle $\theta$ and the reduced height $\hat{h}=h / r$. See zoom in app. Appendix.

simplifications made with the Navier-Stokes equation to obtain the analytical expressions of $k, b$ and $m$. For the second comparison, we directly used experimental data in numerical simulations: due to experimental errors, the symmetry around the plane containing the neck $(z=0)$ was not exactly verified. The numerical simulations were performed with Comsol MulTipHYsics 3.5a.

\subsection{Analytical stiffness}

The relation $r(z)$ (11) was integrated considering the geometrical parameters (radius, height and edge angle). The height was increased by $0.1 \%$, keeping the volume and the radius constant, giving a new curvature and a new edge angle. Then the numerical derivative was performed.

We present these results as $k$-maps. These maps represent the reduced stiffness $\hat{k}=k / \gamma$, according to the edge angle and the reduced height (i.e. form factor) $\hat{h}=h / r$. The construction on the

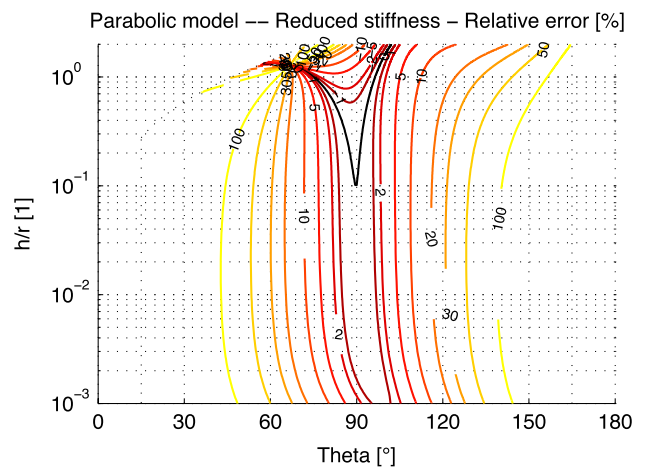

(a) Relative error on the reduced stiffness computed from parabolic model $\left(\hat{k}_{p m}-\hat{k}\right) / \hat{k}$ according to the edge angle $\theta$ and the reduced height $\hat{h}=h / r$. $k$-maps is built on a mesh of uniformly spaced points (600 points for $\theta$ and 2000 points for $\hat{h}$ ). Some extra points were added at small gaps (uniformly logarithmically spaced). Fig. 7 shows the reduced stiffness computed by numerical integration. A more readable map is given in app. Appendix.

The relative errors on the parabolic and circular models are shown in Fig. 8(a) and (b) (computed with (10) and equations from Table 1), and prove that the circular approximation is more accurate. Special care must be used in the evaluation of the relative error near the region where the stiffness is zero: by definition, any small difference of value produces an error tending to infinity. The error tends to zero when the shape approaches a cylinder. The circular model gives an error below 30\% for form factor $\hat{h}<0.1$.

The stiffness coefficient $k$ is not always positive. As illustrated in Fig. 9, when the gap increases, the meniscus curvature decreases, reducing the inner pressure. The second term of the derivative of the force (10) is always positive. On the contrary, the sign of the contribution of the surface tension force, expressed in the first term of (10), is changing: the edge angle always decreases as the gap increases. For angles below $90^{\circ}$ the stiffness is negative, while bigger angles give a positive stiffness.

Fig. 10(a) represents a part of the map of the reduced force $\hat{F}=F / r \gamma$ according to the edge angle $\theta$ and the reduced height $\hat{h}$. The bold line is the evolution of the $\theta$ when the gap increases at constant volume. The evolution of the force along this curve is represented in Fig. 10(b) (plain line). As the gap increases, the force reaches a maximum and begins to decrease. The derivative (i.e., the stiffness) is therefore negative (dashed line).

Consequently, for certain configurations the meniscus can be considered as an anti-spring. Anti-springs are unstable because the force tends to deviate from the equilibrium state. However, if the anti-spring is mechanically constrained - as in the case of a meniscus - it is not unstable: the gap is fixed externally, whatever the force.

\subsection{Analytical and numerical comparison}

The first set of experiments considers a fully-symmetric case: axisymmetry and top-down symmetry. For each model, we compared the gain curves. We plot the asymptotes for each states as:

$$
\begin{aligned}
& \log G_{k}(\omega)=\log k \\
& \log G_{b}(\omega)=\log \omega+\log b \\
& \log G_{m}(\omega)=2 \log \omega+\log m .
\end{aligned}
$$

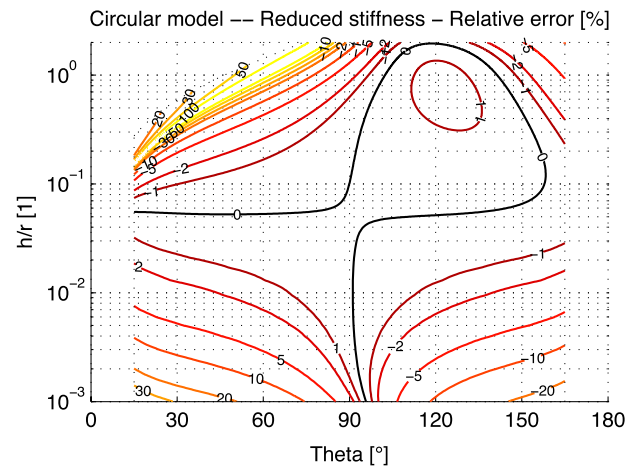

(b) Relative error on the reduced stiffness computed from circular model $\left(\hat{k}_{c m}-\hat{k}\right) / \hat{k}$ according to the edge angle $\theta$ and the reduced height $\hat{h}=h / r$.

Fig. 8. Relative error for analytical models in logarithmic scale. 


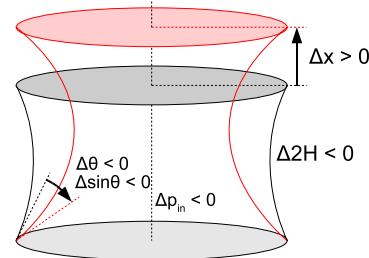

(a) When $\theta<90^{\circ}$, the Laplace force increases while the surface tension force decreases.

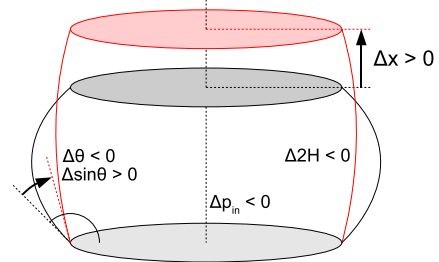

(b) When $\theta>90^{\circ}$, both the Laplace force and the surface tension force increase.

Fig. 9. Evolution of edge angle and inner pressure as the gap increases at constant volume.

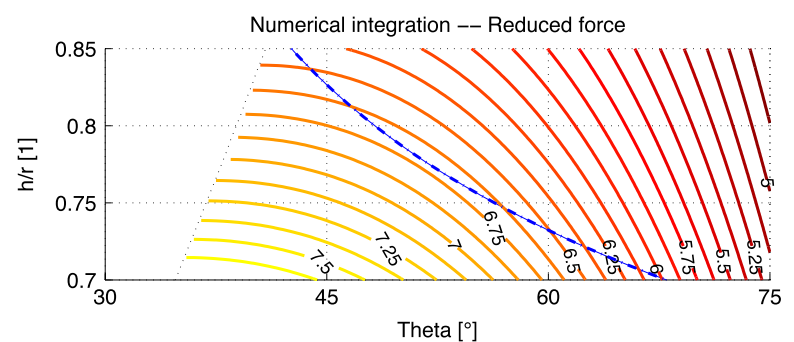

(a) Map of the reduced force $\hat{F}=F / r \gamma$ according to the edge angle $\theta$ and the reduced height $\hat{h}=h / r$. The dashed line is a constant volume line.

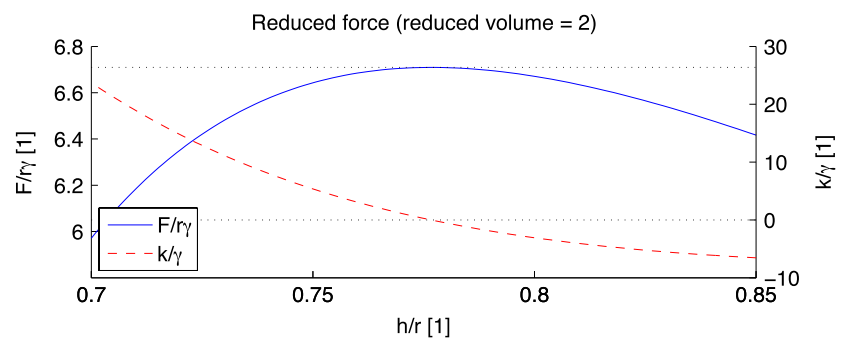

(b) Evolution of the reduced force and reduced stiffness along the constant volume line, in the direction of increasing gap.

Fig. 10. Illustration of origin of negative stiffness.

Table 3

Parameter of numerical simulations.

\begin{tabular}{lll}
\hline Parameter & Symbol & Value \\
\hline Radius & $r_{b}$ & $0.75 \mathrm{~mm}$ \\
Gap & $h$ & $0.15,0.25 \mathrm{~mm}$ \\
Edge angle & $\theta$ & $45^{\circ}, 90^{\circ}, 135^{\circ}$ \\
Viscosity & $\mu$ & $0.001-1 \mathrm{~Pa} \mathrm{~s}$ \\
Surface tension & $\gamma$ & $20 \mathrm{mN} / \mathrm{m}$ \\
Frequency & $f$ & $1 \mathrm{~Hz}$ to $10 \mathrm{kHz}$ \\
\hline
\end{tabular}

The parameters of these simulations are summarised in Table 3. It defines the numerical experimental space studied, made up of all the combinations of the parameters. The parameters chosen cover a wide range of Reynolds numbers and capillary numbers:

$\operatorname{Re}=\frac{\rho f h^{2}}{\mu} 410^{-5} \rightarrow 400$

$\mathrm{Ca}=\frac{\mu f h}{\gamma} 10^{-5} \rightarrow 100$.

The Bode curves (Fig. 11) show that the validity of the assumptions and simplifications made on the Navier-Stokes is acceptable within the range in which the term is not negligible. For example, at very low frequency the estimation of the inertial contribution is erroneous because the assumption on the velocity profile is not valid. However the term is completely negligible with respect to the stiffness.

\subsection{Experimental and numerical comparison}

As already mentioned, during each experiment the geometry of the liquid was controlled by two cameras, before and after the run ( 4 pictures per experiments). Examples of pictures are shown in Fig. 12. The liquid bridge was controlled four times. Geometric measurements are reported in Table A.4 (app. Appendix). Each experiment consists on a frequential scan of 80 points.
Numerical simulations were performed using the parameters of each geometric measurement. Data used are the top and bottom radius, the gap and the top edge angle. The bottom edge angle could not be used for consistency of the initial parameters of the $2 \mathrm{D}$ axisymmetric shape at constant curvature. The Bode curves of representative experiments are shown in Fig. 13.

The results displayed in Fig. 13 are conclusive. All experimental data points are in between the numerical curves. The sign of the stiffness is well predicted, and the data show good repeatability.

However, the variation of the geometric parameters gathered from image analysis produces an important dispersion of the stiffness. The dispersion may be explained for several reasons. First, the positioning error (mainly the tilt in both horizontal directions) gives slightly different profile of the liquid bridge for both cameras. Second, there is a small hysteresis inherent to the piezo actuator. Therefore, the gap and the edge angles may change accordingly. Finally, there is a small amount of liquid that is lost during the experiments, due to flooding outside the pad (in case the pinning was not perfect) or to evaporation.

These geometric errors are less visible on the $b$-state. Indeed, the equivalent damping depends on the volume that is relatively less sensitive to a variation of the free interface. We may see on experiments 11 and 12 (Fig. 13) that for high frequency, the curve goes under the linear asymptote. This is due to the sensor saturation. Consequently, the inertial state could not be observed experimentally because the level of force was too high.

\section{Conclusions and further works}

In this paper, we characterised the behaviour of an axisymmetric liquid bridge under small vertical oscillations. The meniscus was modelled by a Kelvin-Voigt model, consisting of a spring, a damper and a mass in parallel. The paper proposed an abacus for the stiffness, and analytical expressions for the stiffness, the damping and 

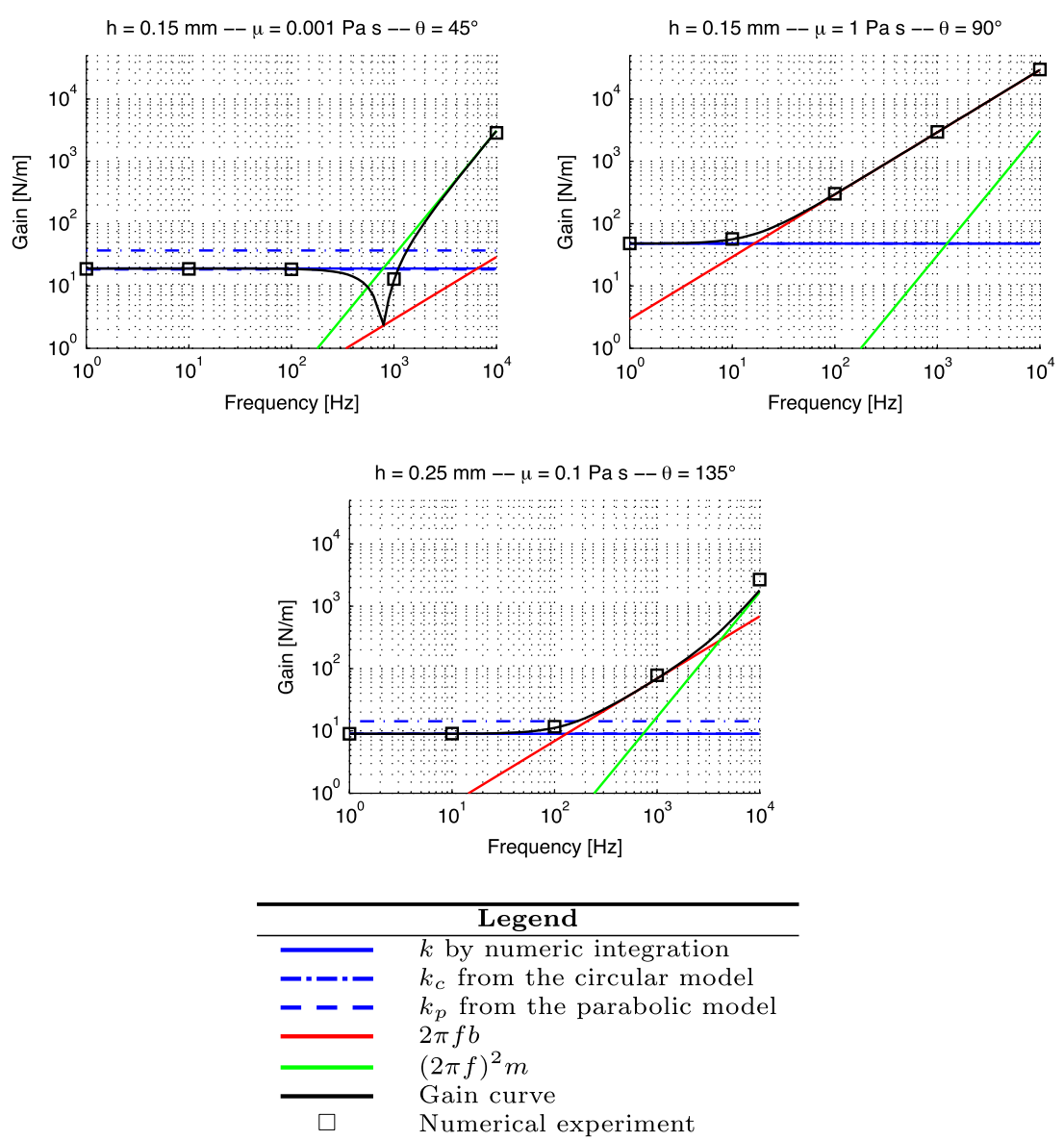

Fig. 11. Gain curves.

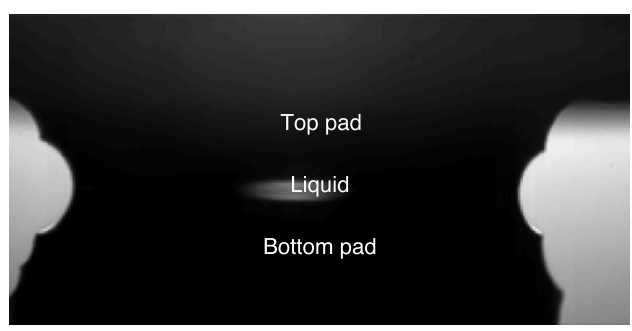

(a) Picture from camera 1 .

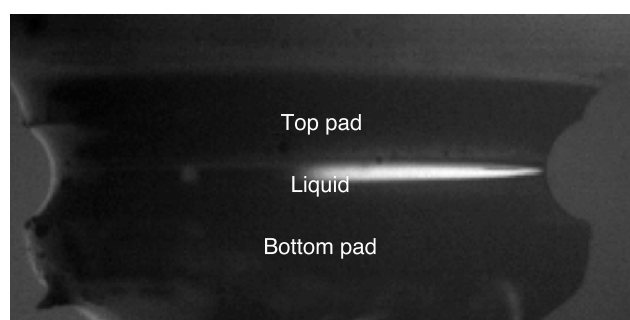

(b) Picture from camera 2.

Fig. 12. Picture from cameras. The diameter of the pad is $1.5 \mathrm{~mm}$.

the inertial coefficients. The validation was provided through numerical simulations and experimental data. The numerical simulations acted as a buffer for results validation: they were compared first with analytical approximations (with a mirror symmetry with respect to the plane containing the neck of the meniscus) and then with experiments.

We showed that it is possible to characterise the meniscus by geometrical and physical parameters of liquids, and without downscaling the system to microscopic dimension. The proposed analytical laws were based on simplifications of the 2D axisymmetric Navier-Stokes equation. They can be used to quickly estimate the order of magnitude of the different parameters $k, b, m$. The first one involves the knowledge of the free liquid surface while the latter are based on the liquid volume. Consequently, the parameter $k$ is more difficult to evaluate: although it is a good approximation, the experimental values of the edge angle and of the curvature of the free surface may be rather difficult to estimate.
The results showed excellent agreement between analytical and numerical models, validating the assumptions on the state of the fluid (static flow for spring state, viscous flow for the damping state and inertial flow for the inertial state). In this case, the characterisation of the inertial effects differs from the Reynolds number since it is usually the convective term $\left(\rho u^{2} / L\right)$ that is considered. In the vibration of the meniscus, the inertial term is related to the acceleration term $(\rho \dot{u})$. Hence an alternative Reynolds number might be defined by the ratio of the inertial forces and the viscous forces:

$\operatorname{Re}_{\text {inertial }}=\frac{\rho V \dot{u}}{\mu u L}=\frac{\rho V \omega}{\mu L}$.

The results showed good agreement between experimental data and numerical simulation, although measurements were difficult to achieve. The present experimental bench did not allow us to inspect the inertial state experimentally since the needed 

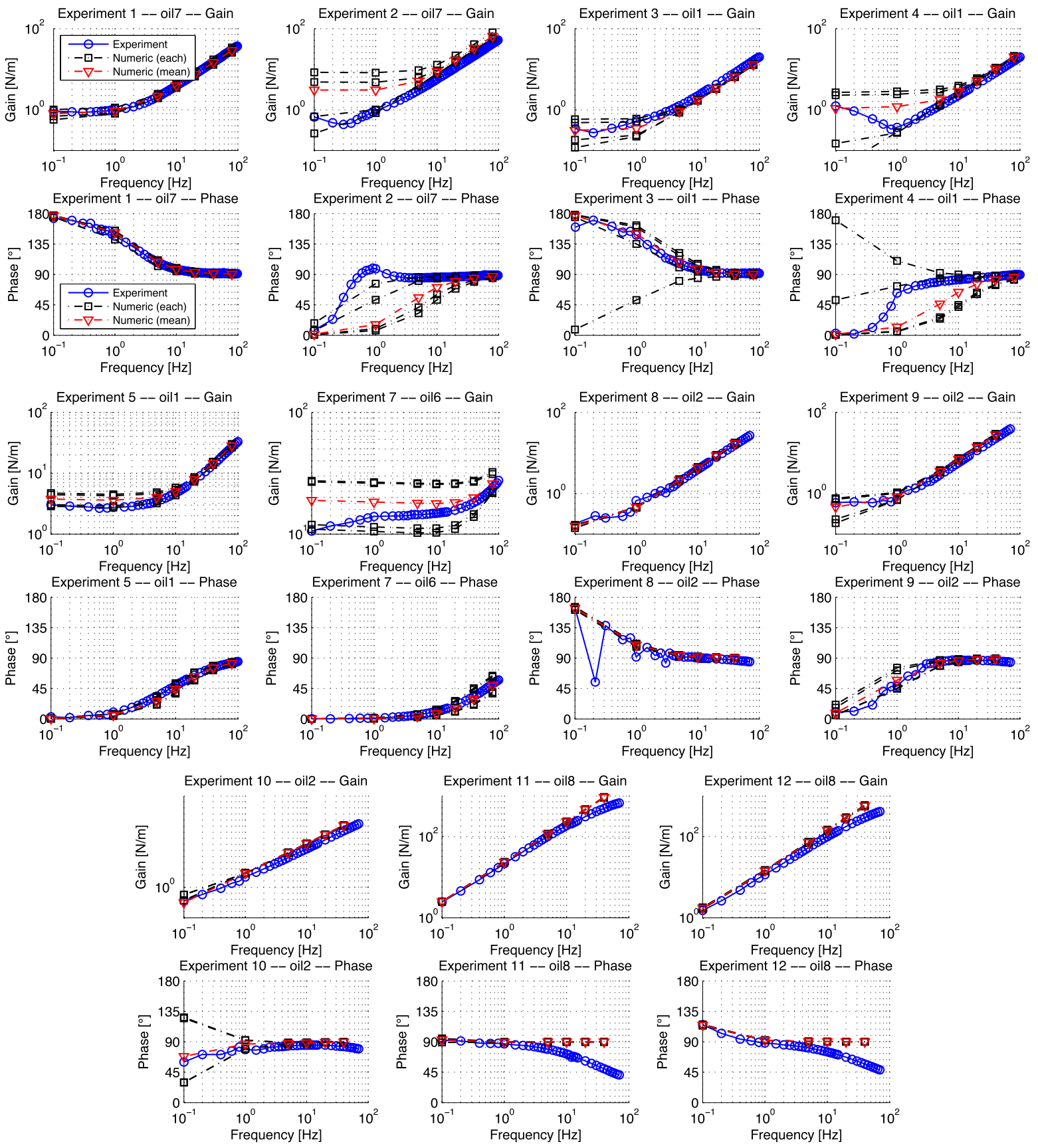

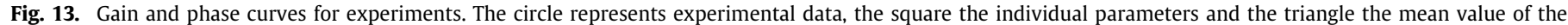
geometric parameters. Experimental parameters are given in Table A.4.

input frequencies are very high and forces generated are too large for the sensor hereby adopted.

Finally, to complete the dynamic description of the liquid bridge investigations of the response along the other degrees of freedom are required. In this respect, we earlier reported the study of the lateral oscillations of the liquid meniscus in [20]. Still, further work is needed.

\section{Acknowledgements}

This work is funded by a grant of the F.R.I.A.-Fonds pour la Formation à la Recherche dans l'Industrie et l'Agriculture. J.-B Valsamis would also like to thank the Laboratory of Molecular and Biomolecular Engineering ${ }^{3}$ (Prof. K. Bartik) for all the subsidiary chemical products, Mr. Zoltan Nagy and Dr. Felix Beyeler from FemtoTool ${ }^{4}$ for their technical support about the force sensor and Mrs Bruno Tartini, Jean-Salvatore Mele and Thierry Delvaux for their technical contribution.

\section{Appendix}

See Fig. 14.

\footnotetext{
3 http://dev.ulb.ac.be/polytech/chimorg/.

4 http://www.femtotools.com/.
} 


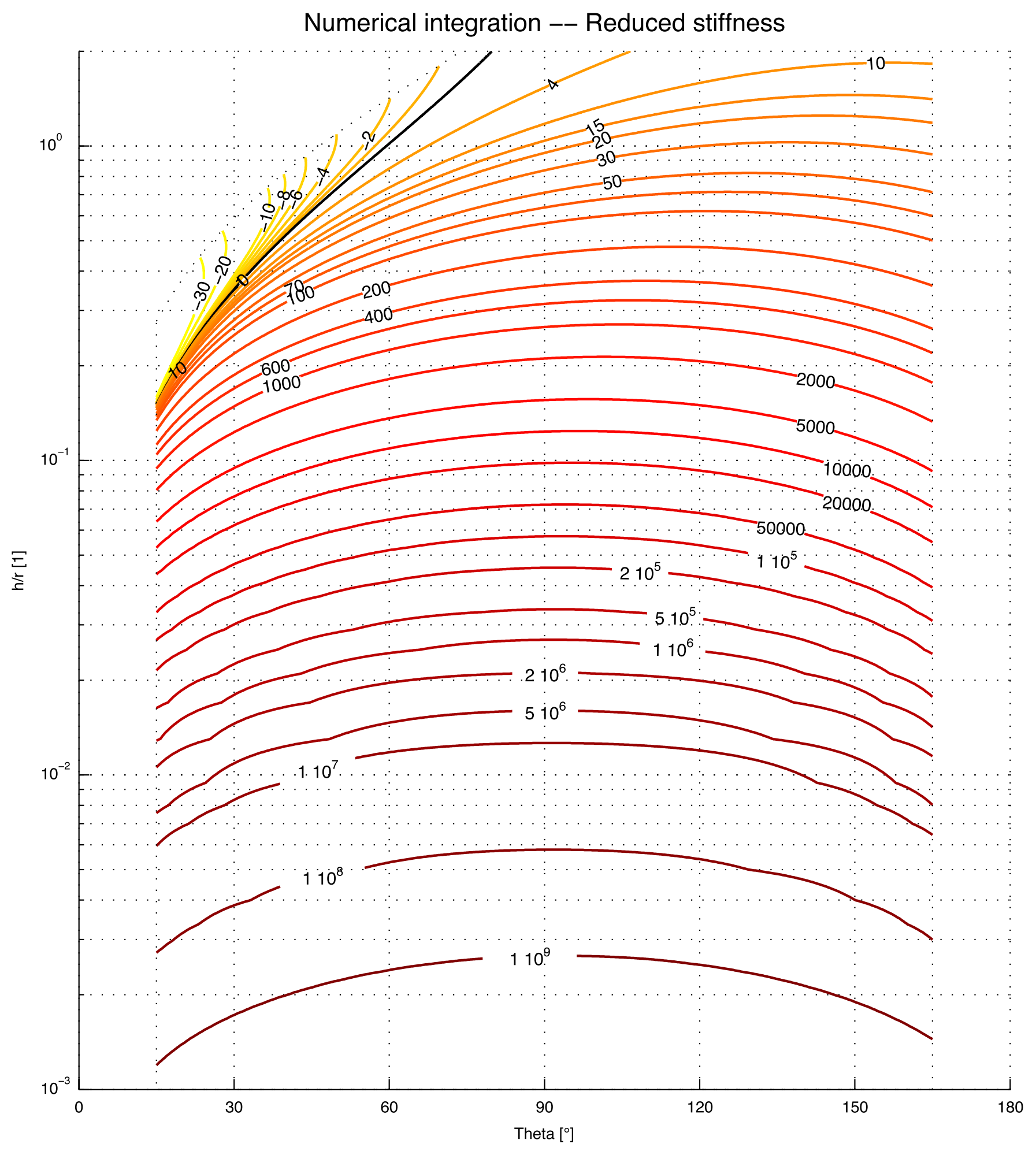

Fig. 14. Big size abacus (Zoom of Fig. 7). 
Table A.4

Experimental results from both cameras. Pictures 1 and 2 have been recorded before the experiment and pictures 3 and 4 have been recorded after.

\begin{tabular}{|c|c|c|c|c|c|c|c|}
\hline Experiment & Picture & $\begin{array}{l}\text { Bottom } \\
\text { radius } \\
{[\mathrm{mm}]}\end{array}$ & $\begin{array}{l}\text { Top } \\
\text { radius } \\
{[\mathrm{mm}]}\end{array}$ & $\begin{array}{l}\text { Bottom } \\
\text { angle } \\
\left.{ }^{\circ}\right]\end{array}$ & $\begin{array}{l}\text { Top } \\
\text { angle } \\
\text { [mm] }\end{array}$ & $\begin{array}{l}\text { Gap } \\
{[\mathrm{mm}]}\end{array}$ & $\begin{array}{l}\text { Volume } \\
{\left[\mathrm{mm}^{3}\right]}\end{array}$ \\
\hline 1 & 1 & 0.717 & 0.696 & 15.9 & 23.6 & 0.284 & 0.354 \\
\hline 1 & 2 & 0.735 & 0.721 & 15.3 & 20.1 & 0.298 & 0.386 \\
\hline 1 & 3 & 0.713 & 0.703 & 18.6 & 22.6 & 0.262 & 0.335 \\
\hline 1 & 4 & 0.738 & 0.732 & 13.9 & 16.2 & 0.281 & 0.374 \\
\hline 1 & Mean & 0.726 & 0.713 & 15.9 & 20.6 & 0.282 & 0.362 \\
\hline 2 & 1 & 0.747 & 0.747 & 43.2 & 43.2 & 0.218 & 0.345 \\
\hline 2 & 2 & 0.77 & 0.746 & 36 & 46.7 & 0.242 & 0.391 \\
\hline 2 & 3 & 0.736 & 0.722 & 18.6 & 24.7 & 0.231 & 0.325 \\
\hline 2 & 4 & 0.747 & 0.729 & 20.2 & 28.1 & 0.235 & 0.342 \\
\hline 2 & Mean & 0.75 & 0.736 & 29.5 & 35.7 & 0.231 & 0.351 \\
\hline 3 & 1 & 0.756 & 0.746 & 28.8 & 32 & 0.323 & 0.473 \\
\hline 3 & 2 & 0.745 & 0.748 & 34.7 & 33.6 & 0.317 & 0.463 \\
\hline 3 & 3 & 0.755 & 0.738 & 21.8 & 27.3 & 0.319 & 0.449 \\
\hline 3 & 4 & 0.767 & 0.755 & 21.4 & 25.2 & 0.322 & 0.468 \\
\hline 3 & Mean & 0.756 & 0.747 & 26.7 & 29.5 & 0.32 & 0.463 \\
\hline 4 & 1 & 0.749 & 0.751 & 45.8 & 45.2 & 0.284 & 0.446 \\
\hline 4 & 2 & 0.755 & 0.752 & 46 & 47.4 & 0.28 & 0.449 \\
\hline 4 & 3 & 0.755 & 0.746 & 24.5 & 27.9 & 0.28 & 0.412 \\
\hline 4 & 4 & 0.758 & 0.735 & 22.4 & 31.1 & 0.273 & 0.4 \\
\hline 4 & Mean & 0.754 & 0.746 & 34.7 & 37.9 & 0.279 & 0.427 \\
\hline 5 & 1 & 0.75 & 0.751 & 50.7 & 50.1 & 0.255 & 0.412 \\
\hline 5 & 2 & 0.76 & 0.752 & 46.5 & 50 & 0.246 & 0.404 \\
\hline 5 & 3 & 0.751 & 0.75 & 41.6 & 42 & 0.251 & 0.396 \\
\hline 5 & 4 & 0.766 & 0.751 & 36.8 & 43.3 & 0.243 & 0.393 \\
\hline 5 & Mean & 0.757 & 0.751 & 43.9 & 46.3 & 0.249 & 0.401 \\
\hline 7 & 1 & 0.743 & 0.747 & 27.4 & 24.9 & 0.158 & 0.243 \\
\hline 7 & 2 & 0.752 & 0.748 & 24.2 & 27.1 & 0.16 & 0.255 \\
\hline 7 & 3 & 0.744 & 0.744 & 40.8 & 40.8 & 0.155 & 0.25 \\
\hline 7 & 4 & 0.758 & 0.752 & 43 & 47.2 & 0.163 & 0.271 \\
\hline 7 & Mean & 0.749 & 0.747 & 33.9 & 35 & 0.159 & 0.255 \\
\hline 8 & 1 & 0.747 & 0.741 & 41 & 42 & 0.554 & 0.743 \\
\hline 8 & 2 & 0.754 & 0.751 & 40.1 & 40.6 & 0.562 & 0.764 \\
\hline 8 & 3 & 0.747 & 0.747 & 40 & 40 & 0.569 & 0.756 \\
\hline 8 & 4 & 0.754 & 0.746 & 39.1 & 40.5 & 0.575 & 0.768 \\
\hline 8 & Mean & 0.75 & 0.746 & 40.1 & 40.8 & 0.565 & 0.758 \\
\hline 9 & 1 & 0.748 & 0.744 & 65.5 & 66.3 & 0.479 & 0.758 \\
\hline 9 & 2 & 0.765 & 0.754 & 65.5 & 67.8 & 0.485 & 0.8 \\
\hline 9 & 3 & 0.745 & 0.744 & 52.1 & 52.3 & 0.482 & 0.714 \\
\hline 9 & 4 & 0.752 & 0.754 & 50.7 & 50.3 & 0.485 & 0.727 \\
\hline 9 & Mean & 0.752 & 0.749 & 58.5 & 59.2 & 0.483 & 0.75 \\
\hline 10 & 1 & 0.743 & 0.75 & 25.4 & 22.4 & 0.26 & 0.381 \\
\hline 10 & 2 & 0.766 & 0.76 & 22.9 & 25.3 & 0.27 & 0.412 \\
\hline 10 & 3 & 0.748 & 0.745 & 29.8 & 30.8 & 0.266 & 0.396 \\
\hline 10 & 4 & 0.748 & 0.754 & 31.6 & 29.5 & 0.269 & 0.406 \\
\hline 10 & Mean & 0.751 & 0.752 & 27.4 & 27 & 0.266 & 0.399 \\
\hline 11 & 1 & 0.734 & 0.751 & 30.5 & 24.6 & 0.293 & 0.422 \\
\hline 11 & 2 & 0.746 & 0.747 & 27.2 & 27.1 & 0.297 & 0.43 \\
\hline 11 & 3 & 0.739 & 0.752 & 32.9 & 28.1 & 0.295 & 0.432 \\
\hline 11 & 4 & 0.755 & 0.75 & 27 & 28.5 & 0.3 & 0.443 \\
\hline 11 & Mean & 0.743 & 0.75 & 29.4 & 27.1 & 0.296 & 0.432 \\
\hline 12 & 1 & 0.753 & 0.757 & 11 & 9.83 & 0.334 & 0.435 \\
\hline 12 & 2 & 0.746 & 0.759 & 16 & 12.4 & 0.343 & 0.451 \\
\hline
\end{tabular}

Table A.4 (continued)

\begin{tabular}{llllllll}
\hline Experiment Picture & $\begin{array}{l}\text { Bottom } \\
\text { radius } \\
{[\mathrm{mm}]}\end{array}$ & $\begin{array}{l}\text { Top } \\
\text { radius } \\
{[\mathrm{mm}]}\end{array}$ & $\begin{array}{l}\text { Bottom } \\
\text { angle } \\
{\left[{ }^{\circ}\right]}\end{array}$ & $\begin{array}{l}\text { Top } \\
\text { angle } \\
{[\mathrm{mm}]}\end{array}$ & $\begin{array}{l}\text { Gap } \\
{[\mathrm{mm}]}\end{array}$ & $\begin{array}{l}\text { Volume } \\
{\left[\mathrm{mm}^{3}\right]}\end{array}$ \\
\hline 12 & 3 & 0.746 & 0.761 & 15.9 & 11.7 & 0.335 & 0.445 \\
12 & 4 & 0.744 & 0.764 & 17.7 & 12.2 & 0.345 & 0.458 \\
12 & Mean & 0.747 & 0.76 & 15.2 & 11.5 & 0.339 & 0.447 \\
\hline
\end{tabular}

\section{References}

[1] P.-G. de Gennes, F. Brochart-Wyard, D. Quéré, Gouttes, bulles, perles et ondes, Belin, 2002.

[2] P. Lambert, Capillary Forces in Microassembly: Modeling, Simulation, Experiments, and Case Study, Microtechnology and MEMS, Springer, 2007.

[3] M. Mastrangeli, J.-B. Valsamis, C.V. Hoof, J.-P. Celis, P. Lambert, Lateral capillary forces of cylindrical fluid menisci: a comprehensive quasi-static study, J. Micromech. Microeng. 20 (7) (2010) http://dx.doi.org/10.1088/09601317/20/7/075041. 075041 (13 p).

[4] W. Lin, S.K. Patra, Y.C. Lee, Design of solder joints for self-aligned optoelectronic assemblies, IEEE Trans. Adv. Packaging 18 (3) (1995) 543-551.

[5] S.K. Patra, Y.C. Lee, Quasi-static modeling of the self-alignment mechanism in flip-chip soldering-part i: single solder joint, J. Electron. Packaging 113 (4) (1991) 337-342.

[6] S.K. Patra, Y.C. Lee, Modeling of self-alignment mechanism in flip-chip soldering-part ii: multichip solder joints, in: Electronic Components and Technology Conference, 1991, Proceedings., 41st, 1991.

[7] M. Mastrangeli, W. Ruythooren, C.V. Hoof, J.-P. Celis, Conformal dip-coating of patterned surfaces for capillary die-to-substrate self-assembly, J. Micromech. Microeng. 19 (4) (2009) 12.

[8] M. Mastrangeli, S. Abbasi, C. Varel, C.V. Hoof, J.-P. Celis, K. Bohringer, Self-assembly from milli- to nanoscales: methods and applications, J Micromech Microeng 19 (8) (2009) 083001. http://dx.doi.org/10.1088/0960$1317 / 19 / 8 / 083001$

[9] K. Brakke, The surface evolver, Exp. Math. 1 (2) (1992) 141-165.

[10] J. Berthier, K. Brakke, F. Grossi, L. Sanchez, L.D. Cioccio, Self-alignment of silicon chips on wafers: a capillary approach, J. Appl. Phys. 108 (2010) 054905.

[11] N. van Veen, Analytical derivation of the self-alignment motion of flip chip soldered components, J. Electron. Packaging 121 (1999) 116-121.

[12] M.-H. Meurisse, M. Querry, Squeeze effects in a flat liquid bridge between parallel solid surfaces, J. Tribology 128 (3) (2006) 575-584.

[13] D. Cheneler, M.C. Ward, M.J. Adams, Z. Zhang, Measurement of dynamic properties of small volumes of fluid using mems, Sens. Actuators, B 130 (2) (2008) 701-706.

[14] J. Engmann, C. Servais, A.S. Burbidge, Squeeze flow theory and applications to rheometry: a review, J. Non-Newtonian Fluid Mech. 132 (1-3) (2005) 1-27.

[15] O. Pitois, P. Moucheront, X. Chateau, Liquid bridge between two moving spheres: an experimental study of viscosity effects, J. Colloid Interface Sci. 231 (1) (2000) 26-31.

[16] N. Boufercha, J. Sägebarth, M. Burgard, N. Othman, D. Schlenker, W. Schäfer, H. Sandmaier, Micro-assembly with fluids, MST/NEWS 2/08, 2008, pp. 29-30.

[17] H. Lu, C. Bailey, Dynamic analysis of flip-chip self alignment, IEEE Trans. Adv. Packaging 28 (3) (2005) 475-480.

[18] J.M. Montanero, Linear dynamics of axisymmetric liquid bridges, Eur. J. Mech. B 22 (2003) 167-178.

[19] J. Montanero, Theoretical analysis of the vibration of axisymmetric liquid bridges of arbitrary shape, Theoret. Comput. Fluid Dynamics 16 (2003) $171-186$.

[20] P. Lambert, M. Mastrangeli, J.-B. Valsamis, G. Degrez, Spectral analysis and experimental study of lateral capillary dynamics (for flip-chip applications), Microfluid Nanofluid. 9 (4-5) (2010) 797-807.

[21] F. Dionnet, Télé-micro-manipulation par adhésion, Ph.D. Thesis, Université Pierre et Marie Curie, Paris, 2005.

[22] V. Vandaele, Contactless handling for micro-assembly: acoustic levitation, Ph.D. Thesis, Université libre de Bruxelles, Brussels, Belgium, 2008. 